\title{
On approximation of the Laplace-Beltrami operator and the Willmore energy of surfaces
}

\author{
Klaus Hildebrandt Konrad Polthier
}

Freie Universität Berlin

\begin{abstract}
Discrete Laplace-Beltrami operators on polyhedral surfaces play an important role for various applications in geometry processing and related areas like physical simulation or computer graphics. While discretizations of the weak Laplace-Beltrami operator are well-studied, less is known about the strong form. We present a principle for constructing strongly consistent discrete Laplace-Beltrami operators based on the cotan weights. The consistency order we obtain, improves previous results reported for the mesh Laplacian. Furthermore, we prove consistency of the discrete Willmore energies corresponding to the discrete Laplace-Beltrami operators.
\end{abstract}

Categories and Subject Descriptors (according to ACM CCS): I.3.5 [Computer Graphics]: Computational Geometry and Object Modeling - Geometric algorithms, languages, and systems

G.1.8 [Numerical Analysis]: Partial Differential Equations-Finite element methods

\section{Introduction}

Discrete Laplace-Beltrami operators on polyhedral surfaces are a key ingredient to various applications in geometry processing, including parametrization, modeling, editing, fairing, shape analysis, interpolation, segmentation, remeshing, compression, and matching [DGSW08, LZ09, RBG*09, SB09, BKP*10]. An important aspect of the construction of discrete differential operators is consistency: does the discrete operator converge to the corresponding continuous operator under suitable refinement? We distinguish between strong and weak consistency, depending on whether the strong form $\left(\Delta: C^{2} \mapsto C^{0}\right)$ or the weak form $\left(\Delta: H_{0}^{1} \mapsto H^{-1}\right)$ of the Laplace-Beltrami operator is approximated.

Whereas the (strong) Laplace-Beltrami operator is defined for Riemannian $C^{2}$-manifolds, its weak form requires less regularity of the manifold. A rigorous definition of the weak Laplace-Beltrami operator on polyhedral surfaces is detailed in [War06]. Discretizing this operator with piecewise-linear finite elements on a polyhedral surface leads to the popular cotan weights [PP93]. Convergence of solutions of the discrete Dirichlet problem was shown by Dziuk [Dzi88] for the case of inscribed polyhedral surfaces and by Hildebrandt et al. [HPW06] in a more general setting. Furthermore, in [HPW06] it was analyzed under what conditions the weak Laplace-Beltrami operators of polyhedral surfaces converge to their counterparts on smooth sur- faces. For many applications, a discretization of the strong Laplace-Beltrami operator is needed, e.g. for approximation of the mean curvature vector. Based on the cotan weights, various constructions of discrete Laplacians have been proposed, see $\left[\mathrm{WBH}^{*} 07, \mathrm{LZ09}, \mathrm{RBG}^{*} 09, \mathrm{BKP}^{*} 10\right]$ and references therein. The cotan Laplacians satisfy structural properties that mimic properties of the continuous operator and are useful for applications, see [DMSB99, WMKG07]. Strong consistency of these operators could only be established for special types of meshes [Xu04] and counter-examples to strong consistency have been reported [Xu04, HPW06].

Recently, Belkin et al. [BSW08] have introduced a strongly consistent discretization of the Laplace-Beltrami operator, called the mesh Laplacian. The construction is based on a discretization of the heat kernel and is related to graph Laplacians used in data analysis and maschine learning [HAvL05, BN08]. The consistency results have been generalized by Belkin et al. [BSW09] to point clouds in $\mathbb{R}^{d}$ and by Dey et al. [DRW10] to convergence of Laplace spectra. The heat kernel signature proposed by Sun et al. [SOG09] is a prominent example of an application of the mesh Laplacian in geometry processing.

The Willmore energy [Wil93] is an nonlinear geometric functional that is used in geometry processing for fairing [HP07, $\left.\mathrm{WBH}^{*} 07\right]$ and hole filling [CDD*04, BS05]. It is closely related to the bending energy of elastic thin plates 
$\left[\mathrm{WBG}^{*} 08\right]$ and the Canham-Helfrich model of thin bilipid membranes [BNSP10]. The Willmore energy of a smooth surface is linked to the Laplace-Beltrami operator, since it equals the $L^{2}$-norm of the Laplacian of the embedding of the surface. To the best of our knowledge, no consistent discrete Willmore energy for polyhedral surfaces (that does not use additional information like quadratically converging surface normals) has been proposed so far.

Contributions. We present a principle for constructing strongly consistent discrete Laplace-Beltrami operators based on the cotan weights. The core of our approach is to test the weak Laplace-Beltrami operator with certain functions that we call $r$-local functions. Among other properties, an $r$-local function at a point $v$ has unit $L^{1}$-norm and its support is contained in the geodesic ball of radius $r$ around $v$. For closely inscribed polyhedral surfaces and interpolating piecewise linear functions, we obtain error estimates in the $L^{\infty}$-norm that depend on $r$ and the mesh size $h$; for convergence one has to choose $r$ asymptotically larger than $h$. Whereas the evaluation of the cotan Laplacians at a vertex requires only the 1-ring, our discrete Laplacians need a region with radius $r$. In this respect, our construction shares similarities with the mesh Laplacian. However, the consistency order we obtain improves the rates reported for the mesh Laplacian. As a direct consequence of the strong consistency of the discrete Laplace-Beltrami operators, we obtain estimates for the pointwise approximation of the mean curvature vector field of a surface. We show consistency of the corresponding discrete Willmore energies, i.e. we prove error estimates for the approximation of the Willmore energy of smooth surfaces by such discrete Willmore energies of polyhedral surfaces.

\section{Laplace-Beltrami Operator on Polyhedral Surfaces}

In this section, we review previous results on the approximation of the Laplace-Beltrami operator and introduce our notation. We consider polyhedral surfaces that approximate a smooth surface in $\mathbb{R}^{3}$, where by a polyhedral surface we mean a finite set of planar triangles in $\mathbb{R}^{3}$ that are glued together in pairs along the edges such that the resulting shape is a two-dimensional manifold. Both surfaces are assumed to be compact, connected, and oriented. We denote smooth surfaces by $M$ and polyhedral surfaces by $M_{h}$, and if we refer to both types of surfaces, we denote the surface by $\mathcal{M}$.

Projection map. To construct a map between $M$ and a neighboring polyhedral surface $M_{h}$, we use the orthogonal projection onto the smooth surface. This map is commonly used, see [Dzi88], [MT04], and [HPW06]. The distance function $\delta_{M}: \mathbb{R}^{3} \mapsto \mathbb{R}_{0}^{+}$is defined as

$$
\delta_{M}(y)=\inf _{x \in M}\|x-y\|_{\mathbb{R}^{3}} .
$$

Since $M$ is compact, for every $y \in \mathbb{R}^{3}$ there is at least one point $x \in M$ that attains the minimum distance to $y$, i.e. $\delta_{M}(y)=\|x-y\|_{\mathbb{R}^{3}}$. Then the straight line passing through $x$ and $y$ meets $M$ orthogonally; thus, $x$ is called an orthogonal projection of $y$ onto $M$. In general, $x$ is not unique by this property. However, there exists an open neighborhood $U_{M}$ of $M$ in $\mathbb{R}^{3}$ such that every point of $U_{M}$ has a unique orthogonal projection onto $M$. The induced projection map $\Pi: U_{M} \mapsto M$ is smooth, a proof of this is contained in a note by Foote [Foo84]. We say that a polyhedral surface $M_{h}$ is inscribed to a smooth surface $M$ if all vertices of $M_{h}$ are on the surface $M$ and that $M_{h}$ is closely inscribed to $M$ if $\Pi_{\mid M_{h}}$, the restriction of $\Pi$ to $M_{h}$, is a bijection. Under these assumptions, the map $\Pi_{\mid M_{h}}$ is bi-Lipschitz, see [War06]. In the following, we assume that $M_{h}$ is closely inscribed to $M$, and, for simplicity, we denote the map $\Pi_{\mid M_{h}}$ by $\pi$.

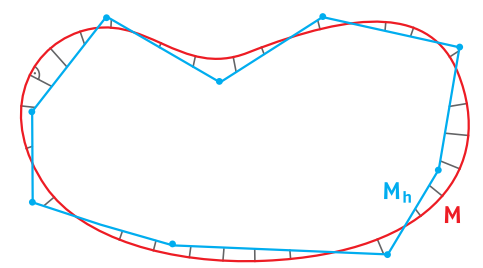

Figure 1: An illustration of the map between the smooth surface and the polyhedral surface is shown.

Metric distortion. The standard scalar product of $\mathbb{R}^{3}$ induces a metric $g$ on $M$ and a metric $g_{h}$ on $M_{h}$. The metric $g$ is Riemannian and $g_{h}$ is flat in the interior of all triangles and edges and has conical singularities at the vertices. To compare the two metrics, we pullback $g_{h}$ to $M$, i.e. we consider the metric

$$
\hat{g}_{h}(X, Y)=g_{h}(\mathrm{~d} \pi X, \mathrm{~d} \pi Y)
$$

on $M$. The metric distortion tensor $A$ measures the distortion between $g$ and $\hat{g}_{h}$. It is uniquely defined (almost everywhere on $M$ ) as the $g$-symmetric tensor field $A$, that satisfies

$$
\hat{g}_{h}(X, Y)=g(A X, Y)
$$

for all smooth vector fields $X, Y$ on $M$. In [HPW06], a closed form representation of $A$ in terms of the curvature of $M$, the distance of corresponding points on $M$ and $M_{h}$, and the deviation of the surface normals at corresponding points on $M$ and $M_{h}$ was derived and used to prove that if a sequence of polyhedral surfaces converges to a smooth surface in the Hausdorff distance, then the metrics of the polyhedral surfaces converge to the metric of the smooth surface if and only if the surface normals converge.

Bounds on the approximation error for various metric properties of $M$ from corresponding properties of $M_{h}$ can be derived from estimates on the metric distortion. The following lemma states three such estimates that were presented in [HPW06, War06]. We start with some notation. For a triangle $T_{h}$ of a polyhedral surface $M_{h}$, let $r_{\text {circ }}\left(T_{h}\right)$ denote the circumradius of $T_{h}$ and let $r_{i n}\left(T_{h}\right)$ denote the inner radius 
of $T_{h}$. We define the mesh size $h$ and the shape regularity $\rho$ of $M_{h}$ as

$$
h=\max _{T_{h} \in M_{h}} r_{\text {circ }}\left(T_{h}\right) \quad \text { and } \quad \rho=\max _{T_{h} \in M_{h}} \frac{r_{\text {circ }}\left(T_{h}\right)}{r_{i n}\left(T_{h}\right)} .
$$

Furthermore, for any $g$-symmetric tensor field $A$, we denote by $\|A\|_{\infty}$ the essential supremum over all $x \in M$ of the absolute values of the eigenvalues of $A$ at $x$.

Lemma 1 Let $M$ be a smooth surface in $\mathbb{R}^{3}$. Then there exists a $h_{0} \in \mathbb{R}^{+}$such that for every polyhedral surface $M_{h}$ that is closely inscribed to $M$ and has mesh size $h<h_{0}$, the estimates

$$
\begin{aligned}
\|A-I d\|_{\infty} & \leq C h^{2}, \\
\|\sqrt{\operatorname{det} A}-1\|_{L^{\infty}} & \leq C h^{2}, \quad \text { and } \\
\left\|\sqrt{\operatorname{det} A} A^{-1}-I d\right\|_{\infty} & \leq C h^{2}
\end{aligned}
$$

hold, where the constant $C$ depends only on $M, h_{0}$, and the shape regularity $\rho$ of $M_{h}$.

The estimates bound the distortion of the metric, the volume form, and, as we will see below, the weak LaplaceBeltrami operators in the operator norm.

Laplace-Beltrami operator. We denote by $L^{p}(\mathcal{M})$, $W^{1, p}(\mathcal{M})$, and $W_{0}^{1, p}(\mathcal{M})$ the Lebesgue and Sobolev spaces on a smooth or polyhedral surface $\mathcal{M}$ and by \|\|$_{L^{p}},\|\|_{W^{1, p}}$, and ||$_{W^{1, p}}$ the corresponding norms and semi-norms. If $p=$ 2, we write $H^{1}(\mathcal{M})=W^{1,2}(\mathcal{M})$ and $H_{0}^{1}(\mathcal{M})=W_{0}^{1,2}(\mathcal{M})$. On a smooth surface $M$, we additionally consider the spaces $C^{k}(M)$ of $k$-times continuously differentiable functions and their norms and semi-norms \|\|$_{C^{k}}$ and ||$_{C^{k}}$, which agree with \|\|$_{W^{k, \infty}}$ and ||$_{W^{k, \infty}}$. For a background on Sobolev spaces on polyhedral surfaces, we refer to [War06]. For simplicity, we denote both the weak and the strong LaplaceBeltrami operator by $\Delta$ and rely on the context to make the distinction. The weak Laplace-Beltrami operator on a smooth or polyhedral surface $\mathcal{M}$ is the continuous linear operator that maps any $u \in H_{0}^{1}(\mathcal{M})$ to the distribution $\Delta u$, which lies in $H^{-1}(\mathcal{M})$, the dual space of $H_{0}^{1}(\mathcal{M})$, and is given by

$$
\langle\Delta u \mid \varphi\rangle=-\int_{\mathcal{M}} g(\nabla u, \nabla \varphi) \mathrm{d} v o l
$$

for all $\varphi \in H_{0}^{1}(\mathcal{M})$. Here $\langle\cdot \mid \cdot\rangle$ denotes the pairing of $H^{-1}(\mathcal{M})$ and $H_{0}^{1}(\mathcal{M})$. The weak Laplacian is a generalization of the (strong or classic) Laplace-Beltrami operator in the sense that for any twice continuously differentiable $u$ on a smooth surface $M$, the (strong) Laplace-Beltrami operator $\Delta u$ of $u$ is the unique continuous function that satisfies

$$
\int_{M} \Delta u \varphi d v o l=-\int_{M} g(\nabla u, \nabla \varphi) \mathrm{d} v o l
$$

for all $\varphi \in H_{0}^{1}(M)$. Using the projection $\pi$, we can pullback any function $u$ defined on $M$ to the function $u \circ \pi$ defined on
$M_{h}$, and for any function $v$ on $M_{h}$, we denote by $\hat{v}$ the function on $M$ that satisfies $\hat{v} \circ \pi=v$. It was shown in [War06] that the pullback of functions induces an isomorphism of the Sobolev spaces $H^{1}(M)$ and $H^{1}\left(M_{h}\right)$. Therefore, to compare $\Delta$ and $\Delta_{h}$, we can pullback $\Delta_{h}$ to $M$ by setting

$$
\left\langle\hat{\Delta}_{h} u \mid \varphi\right\rangle=\left\langle\Delta_{h} u \circ \pi \mid \varphi \circ \pi\right\rangle
$$

for any $\varphi \in H_{0}^{1}(M)$. The operator $\hat{\Delta}_{h}$ is explicitly given by

$$
\left\langle\hat{\Delta}_{h} u \mid \varphi\right\rangle=-\int_{M} g\left(A^{-1} \nabla u, \nabla \varphi\right) \sqrt{\operatorname{det} A} \mathrm{~d} v o l,
$$

and the distance of $\Delta$ and $\hat{\Delta}_{h}$ in the norm of the space of continuous linear operators from $H_{0}^{1}(M)$ to $H^{-1}(M)$ is bounded above by

$$
\left\|\Delta-\hat{\Delta}_{h}\right\|_{O p}=\sup _{u, \varphi}\left|\left\langle\left(\Delta-\hat{\Delta}_{h}\right) u \mid \varphi\right\rangle\right| \leq\left\|\sqrt{\operatorname{det} A} A^{-1}-I d\right\|_{\infty},
$$

where the supremum is taken over all $u, \varphi \in H_{0}^{1}(M)$ with $|u|_{H^{1}}=|\varphi|_{H^{1}}=1$. Then Lemma 1 implies that $\left\|\Delta-\hat{\Delta}_{h}\right\|_{O p}$ is $\mathcal{O}\left(h^{2}\right)$. Proofs can be found in [HPW06, War06].

Piecewise linear functions. Let $S_{h}$ denote the finite dimensional subspace of $H^{1}\left(M_{h}\right)$ consisting of all continuous functions on $M_{h}$ that are linear in each triangle of $M_{h}$, and let $\hat{S}_{h}$ be the space that contains any function $\hat{v} \in H^{1}(M)$ that is a pullback of a function $v$ in $S_{h}$. For any continuous function $u$ on $M$, there are unique functions $u_{h} \in S_{h}$ and $\hat{u}_{h} \in \hat{S}_{h}$ that interpolate $u$ at the vertices of $M_{h}$. The following lemma provides an estimate for the interpolation error that we will use in the next section.

Lemma 2 Let $u \in C^{2}(M)$ and let $\hat{u}_{h} \in \hat{S}_{h}$ be the interpolant of $u$. Then

$$
\left\|\nabla\left(u-\hat{u}_{h}\right)\right\|_{L^{\infty}} \leq C h\left(|u|_{C^{2}}+h|u|_{C^{1}}\right),
$$

where the constant $C$ depends only on $M$ and the shape regularity of $M_{h}$.

Proof Consider a triangle $T_{h}$ of $M_{h}$ and let $T \subset M$ be the projection of $T_{h}$ onto $M$. Then $u \circ \pi_{\mid T_{h}}$ is in $C^{2}\left(T_{h}\right)$ and $u_{h \mid T_{h}}$ is the linear function that interpolates $u$ at the vertices of $T_{h}$. Since $T_{h}$ is a flat triangle, we can use standard estimates for the approximation error

$$
\left\|\nabla_{h}\left(u \circ \pi-u_{h}\right)\right\|_{L^{\infty}\left(T_{h}\right)} \leq C h|u \circ \pi|_{C^{2}\left(T_{h}\right)} .
$$

Furthermore,

$$
\begin{aligned}
& \left\|\nabla\left(u-\hat{u}_{h}\right)\right\|_{L^{\infty}(T)} \\
& \leq\|A\|_{\infty}^{-\frac{1}{2}}\left\|\sqrt{g\left(A^{-1} \nabla\left(u-\hat{u}_{h}\right), \nabla\left(u-\hat{u}_{h}\right)\right)}\right\|_{L^{\infty}(T)} \\
& =\|A\|_{\infty}^{-\frac{1}{2}}\left\|\nabla_{h}\left(u \circ \pi-u_{h}\right)\right\|_{L^{\infty}\left(T_{h}\right)}
\end{aligned}
$$

and it can be shown, see [Dzi88, Lemma 3] and [War06, Lemma 3.3.1], that

$$
|u \circ \pi|_{C^{2}\left(T_{h}\right)} \leq C\left(|u|_{C^{2}(T)}+h|u|_{C^{1}(T)}\right) .
$$

Since the estimates hold for all triangles of $M_{h}$, we have verified (6). 


\section{Pointwise Approximation}

The core of our approach to obtain pointwise approximation estimates is to test the weak Laplace-Beltrami operator with functions whose support gets more and more localized while their $L^{1}$-norm remains constant and the growth of the $W^{1,1}$ norm is bounded. We define:

Definition 3 Let $\mathcal{M}$ be a smooth or a polyhedral surface in $\mathbb{R}^{3}$, and let $C_{D}$ be a positive constant. For any $x \in \mathcal{M}$ and $r \in \mathbb{R}^{+}$, we call a function $\varphi: \mathcal{M} \mapsto \mathbb{R} r$-local at $x$ (with respect to $C_{D}$ ) if the criteria

$$
\begin{array}{ll}
\text { (D1) } & \varphi \in H_{0}^{1}(\mathcal{M}), \\
\text { (D2) } & \varphi(y) \geq 0 \text { for all } y \in \mathcal{M}, \\
\text { (D3) } & \varphi(y)=0 \text { for all } y \in \mathcal{M} \text { with } d_{\mathcal{M}}(x, y) \geq r, \\
\text { (D4) } & \|\varphi\|_{L^{1}}=1 \text {, and } \\
\text { (D5) } & |\varphi|_{W^{1,1}} \leq \frac{C_{D}}{r}
\end{array}
$$

are satisfied.

Functions satisfying properties (D2), (D3), and (D4) can be used to approximate the function value at $x$ of any continuous function $f$ through the integral $\int_{M} f \varphi \mathrm{d} v o l$. In this sense, $r$-local functions are approximations of the delta distribution.

Lemma 4 Let $\varphi \in L^{1}(M)$ satisfy properties (D2), (D3), and (D4) of Definition 3 for some $x \in M$ and $r \in \mathbb{R}^{+}$, and let $f \in C^{1}(M)$. Then, the estimate

$$
\left|f(x)-\int_{M} f \varphi \mathrm{d} v o l\right| \leq\|\nabla f\|_{L^{\infty}} r
$$

holds.

Proof Since $\varphi$ is non-negative and has a unit $L^{1}$-norm, we have

$$
\begin{aligned}
\left|f(x)-\int_{M} f \varphi \mathrm{d} v o l\right| & =\left|\int_{M}(f(x)-f) \varphi \mathrm{d} v o l\right| \\
& \leq \sup _{y \in B_{r}(x)}|f(x)-f(y)| .
\end{aligned}
$$

For any $y$ in the geodesic ball $B_{r}(x)$ around $x$, let $\gamma$ be a (unitspeed parametrized) minimizing geodesic that connects $x$ and $y$. Then

$$
\begin{aligned}
& |f(x)-f(y)|=\left|\int_{\gamma} g(\nabla f(\gamma(t)), \dot{\gamma}(t)) \mathrm{d} t\right| \\
& \leq\|\nabla f\|_{L^{\infty}} \text { length }(\gamma) \leq\|\nabla f\|_{L^{\infty}} r .
\end{aligned}
$$

This implies $\sup _{y \in B_{r}(x)}|f(x)-f(y)| \leq\|\nabla f\|_{L^{\infty}} r$, which concludes the proof.

Certain $r$-local functions even exhibit a higher approximation order. There are $r$-local functions $\varphi$ that satisfy

$$
\left|f(x)-\int_{M} f \varphi \mathrm{d} v o l\right| \leq C|f|_{C^{2}} r^{2}
$$

for all $f \in C^{2}(M)$, where $C$ depends only on $M$. We give an example of such a function in the appendix.

Theorem 5 Let $M$ be a smooth surface in $\mathbb{R}^{3}$ and let $u$ be a smooth function on $M$. Then there exists a $h_{0} \in \mathbb{R}^{+}$such that for every pair consisting of a polyhedral surface $M_{h}$ that is closely inscribed to $M$ and satisfies $h<h_{0}$ and a function $\varphi$ that is $r$-local at a point $y \in M_{h}$, the estimate

$$
\left|\Delta u(x)-\left\langle\Delta_{h} u_{h} \mid \varphi\right\rangle\right| \leq C\left(r+\frac{h}{r}\right)
$$

holds, where $u_{h} \in S_{h}\left(M_{h}\right)$ is the interpolant of $u$ and $x=$ $\pi(y)$. If $\hat{\varphi}$ satisfies (8), then we have

$$
\left|\Delta u(x)-\left\langle\Delta_{h} u_{h} \mid \varphi\right\rangle\right| \leq C\left(r^{2}+\frac{h}{r}\right) .
$$

The constants $C$ depend only on $M, u, h_{0}$, the shape regularity $\rho$ of $M_{h}$, and the constant $C_{D}$ of $\varphi$.

Proof The operator $\Delta_{h}$ and the functions $u_{h}$ and $\varphi$ are defined on $M_{h}$. First, we pullback $\Delta_{h}, u_{h}$, and $\varphi$ to $M$, add some zeros, and use the Cauchy-Schwarz inequality to get

$$
\begin{aligned}
& \left|\Delta u(x)-\left\langle\Delta_{h} u_{h} \mid \varphi\right\rangle\right|=\left|\Delta u(x)-\left\langle\hat{\Delta}_{h} \hat{u}_{h} \mid \hat{\varphi}\right\rangle\right| \\
& \leq|\Delta u(x)-\langle\Delta u \mid \hat{\varphi}\rangle|+\left|\left\langle\Delta\left(u-\hat{u}_{h}\right) \mid \hat{\varphi}\right\rangle\right|+\left|\left\langle\left(\Delta-\hat{\Delta}_{h}\right) \hat{u}_{h} \mid \hat{\varphi}\right\rangle\right| .
\end{aligned}
$$

In the following, we derive bounds for each of the three summands of the right-hand side of (11). We start with the first summand. Since the support of the function $\varphi$ is contained in the geodesic ball $B_{r}(y)$, the support of $\hat{\varphi}$ is contained in the $B_{\|A\|_{\infty} r}(x)$. It follows from Lemma 1 that there is a constant $C$, which depends only on $M$ and $h_{0}$, such that $\hat{\varphi}$ satisfies property (D3) for the point $x$ and the radius $C r$. Thus, $\hat{\varphi} /\|\hat{\varphi}\|_{L^{1}}$ satisfies the requirements of Lemma 4 and we get

$$
\begin{aligned}
& |\Delta u(x)-\langle\Delta u \mid \hat{\varphi}\rangle| \\
& \leq\left|\Delta u(x)-\frac{1}{\|\hat{\varphi}\|_{L^{1}}}\langle\Delta u \mid \hat{\varphi}\rangle\right|+\left|\left(1-\|\hat{\varphi}\|_{L^{1}}\right) \frac{1}{\|\hat{\varphi}\|_{L^{1}}}\langle\Delta u \mid \hat{\varphi}\rangle\right| \\
& \leq C\left(r+h^{2}\right) .
\end{aligned}
$$

Here, we use Lemma 4 and the estimate

$$
\left|\|\hat{\varphi}\|_{L^{1}}-1\right| \leq C h^{2},
$$

which follows from Lemma 1 , in the last step. If $\hat{\varphi}$ satisfies (8), then using (8) instead of Lemma 4 yields

$$
|\Delta u(x)-\langle\Delta u \mid \hat{\varphi}\rangle| \leq C\left(r^{2}+h^{2}\right) .
$$

To establish a bound on the second summand we show that the bound $\left\|\nabla_{h} \varphi\right\|_{L^{1}\left(M_{h}\right)} \leq C \frac{1}{r}$ holds for $\|\nabla \hat{\varphi}\|_{L^{1}}$ as well

$$
\begin{aligned}
& \|\nabla \hat{\varphi}\|_{L^{1}}=\int_{M}\|\nabla \hat{\varphi}\|_{g} \mathrm{~d} v o l \\
& \leq\left\|\operatorname{det} A A^{-1}\right\|_{\infty}^{-\frac{1}{2}} \int_{M} \sqrt{g\left(A^{-1} \nabla \hat{\varphi}, \nabla \hat{\varphi}\right)} \sqrt{\operatorname{det} A} \mathrm{~d} v o l \\
& =\left\|\operatorname{det} A A^{-1}\right\|_{\infty}^{-\frac{1}{2}}\left\|\nabla_{h} \varphi\right\|_{L^{1}\left(M_{h}\right)} \\
& \leq C \frac{1}{r} .
\end{aligned}
$$

(C) 2011 The Author(s) (C) 2011 The Eurographics Association and Blackwell Publishing Ltd. 
Then, we apply Hölder's inequality and Lemma 2 to get

$$
\begin{aligned}
& \left|\left\langle\Delta\left(u-\hat{u}_{h}\right) \mid \hat{\varphi}\right\rangle\right|=\left|\int_{M} g\left(\nabla\left(u-\hat{u}_{h}\right), \nabla \hat{\varphi}\right) \mathrm{d} v o l\right| \\
& \leq\left\|\nabla\left(u-\hat{u}_{h}\right)\right\|_{L^{\infty}}\|\nabla \hat{\varphi}\|_{L^{1}} \leq C \frac{h}{r} .
\end{aligned}
$$

A bound on the third summand follows from estimate (4) of Lemma 1 and Lemma 2

$$
\begin{aligned}
& \left|\left\langle\left(\Delta-\hat{\Delta}_{h}\right) \hat{u}_{h} \mid \hat{\varphi}\right\rangle\right| \\
& =\int_{M} g\left(\left(I d-\sqrt{\operatorname{det} A} A^{-1}\right) \nabla \hat{u}_{h}, \nabla \hat{\varphi}\right) \mathrm{d} v o l \\
& \leq\left\|I d-\sqrt{\operatorname{det} A} A^{-1}\right\|_{\infty}\left\|\nabla \hat{u}_{h}\right\|_{L^{\infty}}\|\nabla \hat{\varphi}\|_{L^{1}} \\
& \leq C \frac{h^{2}}{r} .
\end{aligned}
$$

This bound is quadratic in $h$ and therefore small compared to the bound on the second summand. The combination of the bounds on the three summands completes the proof.

The theorem is stated in a general setting that assumes no correlation of $r$ and $h$. The following corollary shows how to choose $r$ to get the optimal approximation order in $h$.

Corollary 6 Under the assumptions of Theorem 5, if $r=\sqrt{h}$, then

$$
\left|\Delta u(x)-\left\langle\Delta_{h} u_{h} \mid \varphi\right\rangle\right| \leq C \sqrt{h},
$$

and if $\hat{\varphi}$ satisfies (8) and $r=h^{\frac{1}{3}}$, then

$$
\left|\Delta u(x)-\left\langle\Delta_{h} u_{h} \mid \varphi\right\rangle\right| \leq C h^{\frac{2}{3}} .
$$

\section{Discrete Laplace-Beltrami Operators}

Based on the approximation results of the last section, we construct discrete Laplace-Beltrami operators and prove strong consistency. Then, we discuss matrix representations of the operators and compare the consistency rates of our approach and the mesh Laplacian.

Let $n$ be the number of vertices of $M_{h}$ and let $\left\{v_{1}, v_{2}, \ldots, v_{n}\right\}$ denote the set of vertices. Any function $u_{h} \in$ $S_{h}\left(M_{h}\right)$ is uniquely determined by its function values at the vertices. The vector $\left(u_{h}\left(v_{1}\right), u_{h}\left(v_{2}\right), \ldots, u_{h}\left(v_{n}\right)\right)$ is called the nodal vector, and we shall describe discrete LaplaceBeltrami operators by their action on nodal vectors. Let $\left\{\varphi_{i}\right\}_{i \in\{1,2, \ldots n\}}$ be a set of functions such that every $\varphi_{i}$ is $r$-local at the vertex $v_{i} \in M_{h}$. Then, we define the discrete Laplace-Beltrami operator $\Delta_{h}^{\left\{\varphi_{i}\right\}}$ associated to $\left\{\varphi_{i}\right\}$ as

$$
\begin{gathered}
\Delta_{h}^{\left\{\varphi_{i}\right\}}: S_{h} \mapsto S_{h} \\
\left(\begin{array}{c}
u_{h}\left(v_{1}\right) \\
u_{h}\left(v_{2}\right) \\
\ldots \\
u_{h}\left(v_{n}\right)
\end{array}\right) \mapsto\left(\begin{array}{c}
\left\langle\Delta_{h} u_{h} \mid \varphi_{1}\right\rangle \\
\left\langle\Delta_{h} u_{h} \mid \varphi_{2}\right\rangle \\
\cdots \\
\left\langle\Delta_{h} u_{h} \mid \varphi_{n}\right\rangle
\end{array}\right) .
\end{gathered}
$$

(c) 2011 The Author(s)

(c) 2011 The Eurographics Association and Blackwell Publishing Ltd.
For each $\varphi_{i}$ there is a constant $C_{D, i}$ such that (D5) of Definition 3 is satisfied. In the following, we refer to the maximum of the $C_{D, i}$ as the constant $C_{D}$ of $\left\{\varphi_{i}\right\}$.

Theorem 7 Let $M$ be a smooth surface in $\mathbb{R}^{3}$, and let $u$ be a smooth function on $M$. Then there exists a $h_{0} \in \mathbb{R}^{+}$such that for every pair consisting of a polyhedral surface $M_{h}$ that is closely inscribed to $M$ and satisfies $h<h_{0}$ and a set of functions $\left\{\varphi_{i}\right\}_{i \in\{1,2, . . n\}}$ such that every $\varphi_{i}$ is $r$-local at the vertex $v_{i} \in M_{h}$ with $r=\sqrt{h}$, the estimate

$$
\sup _{y \in M_{h}}\left|\Delta u(\pi(y))-\Delta_{h}^{\left\{\varphi_{i}\right\}} u_{h}(y)\right| \leq C \sqrt{h}
$$

holds, where $u_{h} \in S_{h}\left(M_{h}\right)$ is the interpolant of $u$. If every $\hat{\varphi}_{i}$ satisfies (8) and $r=h^{\frac{1}{3}}$, then we have

$$
\sup _{y \in M_{h}}\left|\Delta u(\pi(y))-\Delta_{h}^{\left\{\varphi_{i}\right\}} u_{h}(y)\right| \leq C h^{\frac{2}{3}} .
$$

The constants $C$ depend only on $M, u, h_{0}$, the shape regularity $\rho$ of $M_{h}$, and the constant $C_{D}$ of $\left\{\varphi_{i}\right\}$.

Proof Let $v \in S_{h}$ and $\hat{v} \in \hat{S}_{h}$ be the interpolants on $M_{h}$ and $M$ of the function $\Delta u$. Then the approximation error satisfies (analogous to Lemma 2)

$$
\|\Delta u-\hat{v}\|_{L^{\infty}} \leq C h^{2}\left(|\Delta u|_{C^{2}}+h|\Delta u|_{C^{1}}\right) .
$$

Theorem 5 implies

$$
\left|\Delta u\left(\pi\left(v_{i}\right)\right)-\left\langle\Delta_{h} u_{h} \mid \varphi_{i}\right\rangle\right| \leq C \sqrt{h}
$$

for all $i$. Since $v\left(v_{i}\right)=\hat{v}\left(v_{i}\right)=\Delta u\left(\pi\left(v_{i}\right)\right)$, we have

$$
\left\|v-\Delta_{h}^{\left\{\varphi_{i}\right\}} u_{h}\right\|_{L^{\infty}} \leq C \sqrt{h} .
$$

Combining (14) and (16) shows (12). The proof of the second estimate proceeds analogously.

Matrix representation. We show how the cotan matrix can be used to construct the matrix representation $L$ of $\Delta_{h}^{\left\{\varphi_{i}\right\}}$ with respect to the nodal basis. For simplicity, we assume that the $\varphi_{i}$ are functions in $S_{h}$. Let $S$ denote the cotan matrix, see [PP93], and let $\Phi$ be the matrix with entries $\Phi_{i j}=\varphi_{i}\left(v_{j}\right)$. Then $L$ is the sparse matrix given by

$$
L=-\Phi S \text {. }
$$

The number of entries of $L$ depends on the number of vertices that are in the support of the functions $\varphi_{i}$. When comparing (17) to the construction of cotan Laplacians (as described in $\left[\mathrm{WBG}^{*} 08\right]$ ), we see that $\Phi$ takes the role of the inverse mass matrix.

In general, the matrix $\Phi$ is not symmetric. But, $\Phi$ that are symmetric can be constructed. The matrix can be decomposed into a symmetric and an antisymmetric part

$$
\Phi=\Phi^{\text {sym }}+\Phi^{a s y m}=\frac{1}{2}\left(\Phi+\Phi^{T}\right)+\frac{1}{2}\left(\Phi-\Phi^{T}\right),
$$

where $\Phi^{T}$ denotes the transpose of $\Phi$. For certain choices of $r$-local functions $\left\{\varphi_{i}\right\}$, the entries of $\Phi^{a s y m}$ are small compared to the entries of $\Phi^{\text {sym }}$. For example, if we use the 
geodesic or extrinsic hat functions (see appendix) with the same value of $r$ at all vertices. Then, it is justified to use

$$
L=-\Phi^{s y m} S,
$$

instead of (17).

Comparison with the mesh Laplacian. Bounds on the consistency error of the mesh Laplacian depend on the mesh size $h$, the maximum angle $\eta$ (between normals of the smooth and the polyhedral surface), and a parameter $t$, which controls the width of the heat kernels. The parameter $t$ is the analog of the parameter $r$ in our approach. Under the assumption that the shape regularity of the polyhedral surface is bounded, $\eta=\mathcal{O}(h)$, which is optimal. Then, the consistency order depends on $h$ and $t$. In [BSW09], the bound $\mathcal{O}\left(\frac{h}{t^{2}}+t^{\frac{1}{2}}\right)$ on the consistency error of the mesh Laplacian is derived. The optimal choice $t=h^{\frac{2}{5}}$ yields a consistency order of $h^{\frac{1}{5}}$.

\section{Willmore energy}

The Willmore energy of a smooth surface $M$ in $\mathbb{R}^{3}$ is

$$
W(M)=\int_{M} H^{2} \mathrm{~d} v o l,
$$

where $H$ denotes the mean curvature of $M$. The mean curvature is connected to the Laplace-Beltrami operator by

$$
\mathbf{H}=H N=\Delta I,
$$

where $I$ is the embedding of $M$ in $\mathbb{R}^{3}$ and $N$ is the surface normal field. Then, the Willmore energy of $M$ equals the $L^{2}$-norm of the $\Delta I$. Let $I_{h}: M_{h} \mapsto \mathbb{R}^{3}$ denote the embedding of the polyhedral surface $M_{h}$. Each of the three coordinates of $I_{h}$ is a function in $S_{h}$. Thus, we can define the discrete mean curvature vector associated to a discrete Laplacian $\Delta_{h}^{\left\{\varphi_{i}\right\}}$ analogous to (19) by

$$
\mathbf{H}_{h}^{\left\{\varphi_{i}\right\}}=\Delta_{h}^{\left\{\varphi_{i}\right\}} I_{h} .
$$

If $M_{h}$ is closely inscribed to $M$, Theorem 7 implies

$$
\left.\sup _{y \in M_{h}}\left\|\mathbf{H}(\pi(y))-\mathbf{H}_{h}^{\left\{\varphi_{i}\right\}}(y)\right\|_{\mathbb{R}^{3}} \leq C \sqrt{h} \text { (resp. } C h^{\frac{2}{3}}\right) .
$$

We define the discrete Willmore energy of $M_{h}$ and $\left\{\varphi_{i}\right\}$ analogous to (18) as

$$
W_{h}^{\left\{\varphi_{i}\right\}}\left(M_{h}\right)=\left\|\mathbf{H}_{h}^{\left\{\varphi_{i}\right\}}\right\|_{L^{2}\left(M_{h}\right)}^{2} .
$$

The following theorem shows consistency of the discrete Willmore energies.

Theorem 8 Let $M$ be a smooth surface in $\mathbb{R}^{3}$. Then there exists a $h_{0} \in \mathbb{R}^{+}$such that for every pair consisting of a

\begin{tabular}{|c|c|c|c|c|}
\hline $\mathrm{h}$ & $\left\|\mathbf{H}(x)-\mathbf{H}_{h}^{\varphi} \sqrt[3]{h}\right\|$ & eoc & $\left\|\mathbf{H}(x)-\mathbf{H}_{h}^{\varphi} \sqrt{\sqrt{h}}\right\|$ & eoc \\
\hline 0.9907594 & 0.1473438 & - & 0.1655998 & - \\
\hline 0.4072441 & 0.0718663 & 0.81 & 0.0816863 & 0.79 \\
\hline 0.1376594 & 0.0343882 & 0.68 & 0.0439559 & 0.57 \\
\hline 0.0407502 & 0.0151471 & 0.67 & 0.0238574 & 0.50 \\
\hline 0.0111478 & 0.0063759 & 0.67 & 0.0126259 & 0.49 \\
\hline 0.0042305 & 0.0033417 & 0.67 & 0.0078440 & 0.49 \\
\hline 0.0015209 & 0.0016897 & 0.67 & 0.0047346 & 0.49 \\
\hline $\mathrm{h}$ & $\left\|\mathbf{H}(x)-\mathbf{H}_{h}^{\varphi^{\varphi} \sqrt[3]{h}}\right\|$ & eoc & $\left\|\mathbf{H}(x)-\mathbf{H}_{h}^{\varphi_{h}}\right\|$ & eoc \\
\hline 0.1819730 & 0.1159240 & ב- & 0.0681956 & - \\
\hline 0.0615573 & 0.0469350 & 0.83 & 0.6798970 & -2.10 \\
\hline 0.0182239 & 0.0206826 & 0.67 & 0.0328904 & 2.50 \\
\hline 0.0049855 & 0.0087871 & 0.66 & 0.5920200 & -2.20 \\
\hline 0.0018919 & 0.0045958 & 0.67 & 0.1372740 & 1.50 \\
\hline 0.0006802 & 0.0023197 & 0.67 & 0.0918593 & 0.39 \\
\hline 0.0002655 & 0.0012393 & 0.67 & 0.1451020 & -0.49 \\
\hline
\end{tabular}
polyhedral surface $M_{h}$ that is closely inscribed to $M$ and satisfies $h<h_{0}$ and a set of functions $\left\{\varphi_{i}\right\}_{i \in\{1,2, . . n\}}$ such that every $\varphi_{i}$ is $r$-local at the vertex $v_{i} \in M_{h}$ with $r=\sqrt{h}$, the estimate

$$
\left|W(M)-W_{h}^{\left\{\varphi_{i}\right\}}\left(M_{h}\right)\right| \leq C \sqrt{h}
$$

Table 1: Approximation errors and the experimental orders of convergence for the approximation of the mean curvature vector at a point of a smooth surface are shown.

holds. If every $\hat{\varphi}_{i}$ satisfies (8) and $r=h^{\frac{1}{3}}$, then we have

$$
\left|W(M)-W_{h}^{\left\{\varphi_{i}\right\}}\left(M_{h}\right)\right| \leq C h^{\frac{2}{3}} .
$$

The constants $C$ depend only on $M, h_{0}$, the shape regularity $\rho$ of $M_{h}$, and the constant $C_{D}$ of $\left\{\varphi_{i}\right\}$.

Proof To compare $\mathbf{H}$ and $\mathbf{H}_{h}^{\left\{\varphi_{i}\right\}}$, we consider the vector field $\hat{\mathbf{H}}_{h}^{\left\{\varphi_{i}\right\}} \in \hat{S}_{h}^{3}$ given by $\hat{\mathbf{H}}_{h}^{\left\{\varphi_{i}\right\}} \circ \pi=\mathbf{H}_{h}^{\left\{\varphi_{i}\right\}}$. We split the approximation error in two terms, the first term measures the difference of $\mathbf{H}$ and $\hat{\mathbf{H}}_{h}^{\left\{\varphi_{i}\right\}}$ and the second term measures the difference of the $L^{2}$-norms of $M$ and $M_{h}$ :

$$
\begin{aligned}
& \left|W(M)-W_{h}^{\left\{\varphi_{i}\right\}}\left(M_{h}\right)\right|=\left|\|\mathbf{H}\|_{L^{2}(M)}^{2}-\left\|\mathbf{H}_{h}^{\left\{\varphi_{i}\right\}}\right\|_{L^{2}\left(M_{h}\right)}^{2}\right| \\
& \leq\left|\|\mathbf{H}\|_{L^{2}(M)}^{2}-\left\|\hat{\mathbf{H}}_{h}^{\left\{\varphi_{i}\right\}}\right\|_{L^{2}(M)}^{2}\right|+|| 1-\sqrt{\operatorname{det} A}\left|\left\|\hat{\mathbf{H}}_{h}^{\left\{\varphi_{i}\right\}}\right\|_{L^{2}(M)}^{2}\right| \\
& \leq\left\|\mathbf{H}-\hat{\mathbf{H}}_{h}^{\left\{\varphi_{i}\right\}}\right\|_{L^{\infty}(M)}\left\|\mathbf{H}+\hat{\mathbf{H}}_{h}^{\left\{\varphi_{i}\right\}}\right\|_{L^{1}(M)}+C h^{2}\left\|\hat{\mathbf{H}}_{h}^{\left\{\varphi_{i}\right\}}\right\|_{L^{2}(M)}^{2} .
\end{aligned}
$$

Here, we use Hölder's inequality and Lemma 1 in the last step. By Theorem 7, the term

$$
\left\|\mathbf{H}-\hat{\mathbf{H}}_{h}^{\left\{\varphi_{i}\right\}}\right\|_{L^{\infty}(M)}=\sup _{y \in M_{h}}\left\|\Delta I(\pi(y))-\Delta_{h}^{\left\{\varphi_{i}\right\}} I_{h}(y)\right\|
$$

is bounded by $C \sqrt{h}$, resp. $C h^{\frac{2}{3}}$. Furthermore, Theorem 7 guarantees that there are upper bounds for $\left\|\mathbf{H}+\hat{\mathbf{H}}_{h}^{\left\{\varphi_{i}\right\}}\right\|_{L^{1}(M)}$ and $\left\|\hat{\mathbf{H}}_{h}^{\left\{\varphi_{i}\right\}}\right\|_{L^{2}(M)}^{2}$.

\section{Experiments}

In this section, we show results of experiments concerning the consistency error and the consistency order. For a parametrized surface $M$, we consider inscribed polyhedral surfaces with decreasing mesh size $h$ and approximate the mean curvature vector of $M$ at a point $x \in M$ by $\mathbf{H}_{h}^{\varphi_{r}}=$ $\left\langle\Delta_{h} I_{h} \mid \varphi_{r}\right\rangle$, where $\varphi_{r} \in S_{h}$ is an $r$-local function on $M_{h}$. The 
tables show the approximation error and the experimental order of convergence. Let $e_{h_{i}}$ and $e_{h_{i+1}}$ be the approximation errors of some quantity for the decreasing mesh sizes $h_{i}$ and $h_{i+1}$. Then the experimental order of convergence (eoc) of the quantity is defined as

$$
\operatorname{eoc}\left(h_{i}, h_{i+1}\right)=\log \frac{e_{h_{i}}}{e_{h_{i+1}}}\left(\log \frac{h_{i}}{h_{i+1}}\right)^{-1} .
$$

In the first example, we consider a torus of revolution. The upper part of Table 1 shows the approximation error $\left\|\mathbf{H}(x)-\mathbf{H}_{h}^{\varphi_{r}}\right\|_{\mathbb{R}^{3}}$ obtained with two different types of $r$-local functions and confirms both estimates of Theorem 5 . The first function is (a piecewise linear approximation of) the extrinsic hat function (see appendix). It is given by

$$
\varphi_{r}=\frac{\tilde{\varphi}_{r}}{\left\|\tilde{\varphi}_{r}\right\|_{L^{1}}},
$$

where $\tilde{\varphi}_{r}$ is the function in $S_{h}$ that at any vertex $v \in M_{h}$ takes the value

$$
\tilde{\varphi}_{r}(v)=\max \left\{1-\frac{\|x-v\|_{\mathbb{R}^{3}}}{r}, 0\right\} .
$$

We set $r=h^{\frac{1}{3}}$ and due to the approximate symmetry of $\varphi_{r}$ around $x$, we obtain an eoc of $h^{\frac{2}{3}}$. The second function sets $r=h^{\frac{1}{2}}$, and we disturb the symmetry around $x$ by translating the center of the extrinsic hat function (away from $x$ ) by a random vector of length $\sqrt{h} / 20$. The resulting function does not satisfy (8), and we get the expected eoc of $h^{\frac{1}{2}}$. We would like to remark that in this experiment (and in many other similar settings) we got an eoc of $h$ if we set $r=h^{\frac{1}{2}}$ and and do not translate the center of the function. This suggests the question whether it is possible the improve the consistency order of $h^{\frac{2}{3}}$ for functions that satisfy (8). In the second example, we consider polyhedral surfaces that approximate the sphere, but the vertex positions are corrupted with random noise of order $h^{2}$. The lower part of Table 1 shows that for $r=h^{\frac{1}{3}}$ we still obtain the same eoc, whereas for $r=h$ there is no convergence. In the third example, we approximate the Willmore energy of a torus of revolution by the discrete Willmore energy that we obtain by using functions

\begin{tabular}{|c|c|c|c|}
$\mathrm{h}$ & $W_{h}^{\left\{\varphi_{i}\right\}}\left(M_{h}\right)$ & $W(M)-W_{h}^{\left\{\varphi_{i}\right\}}\left(M_{h}\right)$ & eoc \\
\hline \hline 0.6181260 & 19.8617 & 2.931210 & - \\
0.3542860 & 20.9932 & 1.799680 & 0.88 \\
0.1909680 & 21.7347 & 1.058190 & 0.86 \\
0.0973871 & 22.1986 & 0.594268 & 0.86 \\
0.0491797 & 22.4605 & 0.332392 & 0.85 \\
0.0247127 & 22.6066 & 0.186275 & 0.84 \\
0.0123872 & 22.6876 & 0.105310 & 0.83 \\
0.0062014 & 22.7327 & 0.060178 & 0.81
\end{tabular}

Table 2: Results for approximation of the Willmore energy of a torus of revolution by a discrete Willmore energy. of type (20) at each vertex of the polyhedral surface. Table 2 shows the consistency error and the eoc.

\section{Conclusion}

We have shown that using the weak form of the LaplaceBeltrami operator, one can construct discrete LaplaceBeltrami operators that are strongly consistent. The approximation results are formulated for closely inscribed meshes and therefore depend on the mesh size and the shape regularity. However, the results could be transferred to the more general setting used in [HPW06] that does not restrict vertices to lay on the surface. We think that the presented technique could be helpful for the construction of discrete schemes, based on piecewise linear finite elements, for solving 4th order problems. As a step in this direction, we have proposed a consistent discretization of the Willmore energy.

\section{Acknowledgements}

We would like to thank Ulrich Bauer, Max Wardetzky, and the anonymous reviewers for their valuable comments and feedback. This work was supported by the DFG Research Center MATHEON "Mathematics for Key Technologies" in Berlin.

\section{References}

[BKP*10] Bотsch M., Kobbelt L., Pauly M., Alliez P., LEVy B.: Polygon Mesh Processing. AK Peters, 2010. 1

[BN08] BELKin M., NiYOGi P.: Towards a theoretical foundation for Laplacian-based manifold methods. J. Comput. Syst. Sci. 74 (2008), 1289-1308. 1

[BNSP10] Bonito A., Nochetto R. H., SEBASTIAN PAUletti M.: Parametric FEM for geometric biomembranes. J. Comput. Phys. 229 (May 2010), 3171-3188. 2

[BS05] BOBENKO A. I., SCHRÖDER P.: Discrete Willmore flow. In Proceedings of the ACM SIGGRAPH/Eurographics Symposium on Geometry Processing (2005), pp. 101-110. 1

[BSW08] BELKIN M., SUN J., WANG Y.: Discrete Laplace operator for meshed surfaces. In Symposium on Computational Geometry (2008), pp. 278-287. 1

[BSW09] BELKIN M., SUN J., WANG Y.: Constructing Laplace operator from point clouds in $\mathbb{R}^{d}$. In Proceedings of the twentieth Annual ACM-SIAM Symposium on Discrete Algorithms (2009), SODA '09, pp. 1031-1040. 1, 6

[CDD*04] Clarenz U., Diewald U., Dziuk G., RumpF M., RUSU R.: A finite element method for surface restoration with smooth boundary conditions. Comput. Aided Geom. Des. 21 (2004), 427-445. 1

[DGSW08] Desbrun M., Grinspun E., SChröder P., WARDETZKY M.: Discrete Differential Geometry: An applied introduction. In ACM SIGGRAPH ASIA Courses (2008). 1

[DMSB99] Desbrun M., Meyer M., Schröder P., BARR A. H.: Implicit fairing of irregular meshes using diffusion and curvature flow. Proceedings of ACM SIGGRAPH (1999), 317324. 1 
[DRW10] DEY T. K., RANJAN P., WANG Y.: Convergence, stability, and discrete approximation of Laplace spectra. In Proceedings of the Twenty-First Annual ACM-SIAM Symposium on Discrete Algorithms (2010), SODA '10, SIAM, pp. 650-663. 1

[Dzi88] DzIUK G.: Finite elements for the Beltrami operator on arbitrary surfaces. In Partial Differential Equations and Calculus of Variations (1988), Hildebrandt S., Leis R., (Eds.), Lecture Notes in Mathematics 1357, Springer Verlag. 1, 2, 3

[Foo84] Foote R. L.: Regularity of the distance function. Proceedings of the American Mathematical Society 92, 1 (1984), 153-155. 2

[HAvL05] Hein M., Audibert J.-Y., von LuXburg U.: From graphs to manifolds - weak and strong pointwise consistency of graph Laplacians. In Proceedings of the 18th Conference on Learning Theory (COLT (2005), Springer, pp. 470-485. 1

[HP07] HildebrandT K., POlthier K.: Constraint-based fairing of surface meshes. In Siggraph/Eurographics Sympos. Geom. Processing (2007), pp. 203-212. 1

[HPW06] Hildebrandt K., Polthier K., Wardetzky M.: On the convergence of metric and geometric properties of polyhedral surfaces. Geometricae Dedicata 123 (2006), 89-112. 1, $2,3,7$

[LZ09] LÉVy B., ZHANG H.: Spectral mesh processing. In ACM SIGGRAPH ASIA Courses (2009), pp. 1-47. 1

[MT04] Morvan J.-M., ThiBert B.: Approximation of the normal vector field and the area of a smooth surface. Discrete and Computational Geometry 32, 3 (2004), 383-400. 2

[PP93] Pinkall U., Polthier K.: Computing discrete minimal surfaces and their conjugates. Experim. Math. 2 (1993), 15-36. 1,5

[RBG*09] Reuter M., Biasotti S., Giorgi D., Patanè G., SPAGNUOLO M.: Discrete Laplace-Beltrami operators for shape analysis and segmentation. Computers \& Graphics 33, 3 (2009), 381-390. 1

[SB09] Sorkine O., Botsch M.: Tutorial: Interactive shape modeling and deformation. In Eurographics (2009). 1

[SOG09] Sun J., OvsJanikov M., Guibas L. J.: A concise and provably informative multi-scale signature based on heat diffusion. Computer Graphics Forum 28, 5 (2009), 1383-1392. 1

[War06] WARDETZKY M.: Discrete Differential Operators on Polyhedral Surfaces - Convergence and Approximation. $\mathrm{PhD}$ thesis, Freie Universiät Berlin, 2006. 1, 2, 3

[WBG*08] WARDetzky M., Bergou M., Garg A., HarMON D., ZORIN D., GRINSPUN E.: Simple and efficient implementation of discrete plates and shells. In ACM SIGGRAPH ASIA 2008 courses (2008), pp. 13:1-13:14. 2, 5

[WBH*07] WARDETZKY M., Bergou M., HARMON D., Zorin D., Grinspun E.: Discrete Quadratic Curvature Energies. Computer Aided Geometric Design 24 (2007), 499-518. 1

[Wi193] Willmore T. J.: Riemannian Geometry. Oxford Press, 1993. 1

[WMKG07] WARDETZKY M., MATHUR S., KÄLberer F., GRINSPUN E.: Discrete Laplace operators: No free lunch. In Siggraph/Eurographics Sympos. Geom. Processing (2007), pp. 3337. 1

[Xu04] XU G.: Convergence of discrete Laplace-Beltrami operators over surfaces. Comput. Math. Appl. 48 (2004), 347-360. 1

\section{Appendix}

In this appendix, we discuss a way to construct $r$-local functions. First, we consider a family of $r$-local functions on $\mathbb{R}^{2}$, then we use the Riemannian exponential map to construct a family of $r$-local functions on $M$. Let $\phi \in H^{1}\left(\mathbb{R}^{2}\right)$ be a non-negative function that vanishes in the complement of the open unit ball in $\mathbb{R}^{2}$ and satisfies $\|\phi\|_{L^{1}\left(\mathbb{R}^{2}\right)}=1$. For any $r \in \mathbb{R}^{+}, \phi_{r}$ defined by

$$
\phi_{r}(\cdot)=\frac{1}{r^{2}} \phi\left(\frac{\dot{r}}{r}\right)
$$

is an $r$-local function on $\mathbb{R}^{2}$ and the constant $C_{D}$ equals $|\phi|_{W^{1,1}\left(\mathbb{R}^{2}\right)}$. Since the surface $M$ is compact, the injectivity radius $i(M)$ of $M$ is a strictly positive number. For a point $x \in M$ and an $r \in \mathbb{R}^{+}$, let $B_{r}(x)$ be the open geodesic ball around $x$ in $M$, and let $B_{r}(0)$ denote the open ball of radius $r$ around the origin 0 in $T_{x} M$. The Riemannian exponential map at $x$,

$$
\exp : B_{i(M)}(0) \subset T_{x} M \mapsto M,
$$

is a diffeomorphism of $B_{i(M)}(0)$ and $\exp \left(B_{i(M)}(0)\right)=$ $B_{i(M)}(x)$. Let $\rho \in \mathbb{R}^{+}$be strictly smaller than $i(M)$. Then, for any $r \in(0, \rho), \varphi_{r}$ given by

$$
\varphi_{r}=\left\|\phi_{r} \circ \exp ^{-1}\right\|_{L^{1}(M)}^{-1} \phi_{r} \circ \exp ^{-1}
$$

is $r$-local at $x$. On polyhedral surfaces, we are not interested in $r$-local functions for arbitrarily small $r$, but for $r \sim h^{\frac{1}{2}}$ or $r \sim h^{\frac{1}{3}}$. Furthermore, we restrict our attention to functions in $S_{h}$. The interpolant in $S_{h}$ of an $r$-local function $\varphi_{r}$ on $M$ is an $r$-local function on $M_{h}$, though the values $r$ may differ by $h$.

As an example of this construction of $r$-local functions, let us consider the function $\phi(\cdot)=\frac{3}{\pi} \max \left\{0,1-\|\cdot\|_{\mathbb{R}^{2}}\right\}$ on $\mathbb{R}^{2}$. We call the corresponding functions $\varphi_{r}$ on $M$ geodesic hat functions, since they decay linearly with respect to the geodesic distance to $x$. Explicitly, the functions are given by $\varphi_{r}=\tilde{\varphi}_{r} /\left\|\tilde{\varphi}_{r}\right\|_{L^{1}}$, where $\tilde{\varphi}_{r}(y)=\max \left\{0,1-\delta_{M}(x, y) / r\right\}$ and $\delta_{M}(x, y)$ denotes the geodesic distance of $x$ and $y$. Due to their symmetry around $x$, the functions $\varphi_{r}$ are examples of functions that satisfy the estimate (8). On $M_{h}$ we can construct functions $\varphi_{r, h}$ that approximate $\varphi_{r}$ by replacing the geodesic distance $\delta_{M}$ on $M$ with the geodesic distance $\delta_{M_{h}}$ on $M_{h}$.

In our experiments, we use extrinsic hat functions instead of the geodesic hat functions, i.e. we replace the geodesic distance by the Euclidean distance in $\mathbb{R}^{3}$. For small enough $r$, the extrinsic hat functions satisfy the properties of $r$-local functions, except that we need to modify property $(D 3)$ : the support of the extrinsic hat functions is not contained in $B_{r}(x)$ but there is a constant $C$ depending only on $M$ such that the support is contained in $B_{C r}(x)$. Still, our estimates hold for these functions as well. 